\title{
FIXED POINT INDEX IN HYPERSPACES: A CONLEY-TYPE INDEX FOR DISCRETE SEMIDYNAMICAL SYSTEMS
}

\author{
F. R. RUIZ DEL PORTAL AND J. M. SALAZAR
}

\begin{abstract}
Let $X$ be a locally compact metric absolute neighbourhood retract for metric spaces, $U \subset X$ be an open subset and $f: U \longrightarrow X$ be a continuous map. The aim of the paper is to study the fixed point index of the map that $f$ induces in the hyperspace of $X$. For any compact isolated invariant set, $K \subset U$, this fixed point index produces, in a very natural way, a Conley-type (integer valued) index for $K$. This index is computed and it is shown that it only depends on what is called the attracting part of $K$. The index is used to obtain a characterization of isolating neighbourhoods of compact invariant sets with non-empty attracting part. This index also provides a characterization of compact isolated minimal sets that are attractors.
\end{abstract}

\section{Introduction and preliminary definitions}

The problem of the construction of the Conley index for discrete dynamical systems posed in Conley's book [4] was solved by Robbin and Salamon in [19], using shape theory, for isolated invariant sets of a diffeomorphism on a smooth manifold. Mrozek, in [13], introduced a cohomological Conley index for isolated invariant sets of homeomorphisms. Later, in [14], Mrozek presented a scheme for constructing various Conley indices for locally defined maps that, in particular, extends the previous works.

In order to assign an index to each isolated set, the notion of index pair is needed. The independence of the choice of index pairs to introduce such indices is one of the main themes of the above-mentioned papers.

The reader who is familiar with degree theory will note immediately that Conley indices share similar properties with the fixed point index theory. In this paper we shall construct, in a very general setting, an integer-valued index using the fixed point index of the induced map on the hyperspace of $X$ (endowed with the Hausdorff metric). Our construction is quite natural and from the well-known properties of the fixed point index, the independence of the choices we make for the definition will be obvious. We will not need index pairs to introduce our index but a special class of index pairs will be useful to compute it.

From now onwards $X$ will denote a locally compact, metric absolute neighbourhood retract for metric spaces (ANR). Let us recall some notions that we will need; most of the definitions below have been taken from $[\mathbf{6}, \mathbf{1 4}]$. The reader is referred to $[3,7,18]$ for information about the fixed point index theory in ANRs.

Let $U \subset X$ be an open set. By a (local) semidynamical system we mean a locally defined continuous map $f: U \longrightarrow X$. A function $\sigma: \mathbb{Z} \longrightarrow X$ is said to be a solution to

Received 5 June 2000.

2000 Mathematics Subject Classification 54H20, 54H25.

The first author was supported by the DGES. 
$f$ through $x$ in $N \subset X$ if $f(\sigma(i))=\sigma(i+1)$ for all $i \in \mathbb{Z}, \sigma(0)=x$ and $\sigma(i) \in N$ for all $i \in \mathbb{Z}$. The invariant part of $N, \operatorname{Inv}(N, f)$, is defined as the set of all $x \in N$ that admit a solution to $f$ through $x$ in $N$.

A compact set $K \subset X$ is invariant if $f(K)=K$. An invariant compact set $K$ is isolated with respect to $f$ if there exists a compact neighbourhood $N$ of $K$ such that $\operatorname{Inv}(N, f)=K$. The neighbourhood $N$ is called an isolating neighbourhood of $K$.

Given $X$, we will denote by $2^{X}$ the hyperspace of non-empty compact subsets of $X$ endowed with the Hausdorff metric, $d_{\mathrm{H}}$, defined by

$$
d_{\mathrm{H}}(C, D)=\inf \{\epsilon>0: C \subset B(D, \epsilon) \text { and } D \subset B(C, \epsilon)\}
$$

where $B(K, \epsilon)=\{x \in X: d(x, K)<\epsilon\}$ for any compact set $K \subset X$.

A growth hyperspace $\Lambda$ of $X$ is any closed subspace of $2^{X}$ satisfying the following condition: if $C \in \Lambda$ and $D \in 2^{X}$ are such that $C \subset D$ and every component of $D$ meets $C$, then $D \in \Lambda .2^{X}$ and the hyperspace $C(X)$ of non-empty compact connected subsets of $X$ are growth hyperspaces of $X$.

The following theorem, due to D. W. Curtis, is crucial in our construction.

THEOREM 1 [6, p. 141]. If $X$ is locally continuum-connected (connected and locally continuum-connected), then every growth hyperspace $\Lambda$ of $X$ is an ANR (absolute retract for metric spaces $(A R))$. Conversely, if there exists a growth hyperspace $\Lambda$ such that $C(X) \subset \Lambda$ and $\Lambda$ is an $A N R(A R)$, then $X$ is locally continuum-connected (connected and locally continuum-connected).

A semidynamical system $f: U \longrightarrow X$ induces in a natural way another one $2^{f}: 2^{U} \longrightarrow 2^{X}$.

Let $K \subset U$ be a compact isolated invariant set. Let $N$ be any isolating neighbourhood of $K$. Consider an open set $W$ such that $K \subset W \subset N$. Take $\left.2^{f}\right|_{2^{W}}: 2^{W} \longrightarrow 2^{X}$. It is clear that $\operatorname{Fix}\left(\left.2^{f}\right|_{2^{w}}\right) \subset 2^{K}$, then Fix $\left(\left.2^{f}\right|_{2^{w}}\right)$ is a compact subset of $2^{W}$. On the other hand, $\left.2^{f}\right|_{2^{W}}$ is a compact map because it admits an obvious extension to $2^{N}$. Therefore the fixed point index of $\left.2^{f}\right|_{2^{W}}$ in $2^{X}, i_{2^{X}}\left(\left.2^{f}\right|_{2^{W}}, 2^{W}\right)$, is well defined.

Definition 1. We define the fixed compact index of the pair $(K, f)$ as

$$
I_{X}(K, f)=i_{2} x\left(\left.2^{f}\right|_{2^{w}}, 2^{W}\right) .
$$

REMARK 1. From the excision property of the fixed point index we obtain that $I_{X}(K, f)$ does not depend on the choice of the isolating neighbourhood $N$ of $K$ and the open set $W$.

REMARK 2. It is easy to construct examples where $I_{X}(K, f) \neq i_{X}(f, W)$. In fact, if we consider any flow $\pi$ in $\mathbb{R}^{2}$ with an attractor $K$ which is a closed orbit, we can take $t>0$ such that the period of $x \in K$ is not a multiple of $t$. Let $N \in$ ANR be an isolating neighbourhood of $K$. If $f=\pi_{t}$ it follows that $i_{\mathbb{R}^{2}}(f, W)=0$ because the set of fixed points is empty but using Theorem $1,2^{N}$ is an $\operatorname{AR}\left(2^{N}\right.$ is homeomorphic to the Hilbert cube, see [5] for example), then $I_{\mathbb{R}^{2}}(K, f)=1$ (see Theorem 3).

REMARK 3. If $X$ is not locally compact we still can introduce our index if we assume $f$ to be compact. In this sense, the Rybakowski condition, see $[\mathbf{1 2}, \mathbf{1 3}, \mathbf{1 5}]$, is introduced to define the Conley index when the local compactness of $X$ is not required. 
Remark 4. Note that similar indices can be defined if we restrict ourselves to a particular growth hyperspace of $X$. In the last part of the paper we will present the results we can obtain if we consider the growth hyperspace $C_{m}(X)$ of the non-empty compact subsets of $X$ with at most $m$ connected components.

On the other hand, using the fact that the spaces $F_{k}(X)$ of all non-empty subsets of $X$ consisting of at most $k$ points, are also ANRs (see [16]) we can introduce indices which would measure the periodic points of $f$. In a forthcoming paper we will give a detailed study of these indices.

Definition 2. Let $f: U \subset X \longrightarrow X$ be a semidynamical system. A compact isolated invariant set $K \subset U$ is said to be an attractor if there exists an open neighbourhood $U_{0} \subset U$ of $K$ such that:

(i) $f^{m}\left(U_{0}\right) \subset U$ for every $m \geqslant 1$.

(ii) For every open neighbourhood $V$ of $K$ there is $m(V) \in \mathbb{Z}$ such that $f^{n}\left(U_{0}\right) \subset V$ for all $n \geqslant m(V)$.

When computing the classical fixed point index of a map $f: W \subset \mathbb{R}^{n} \longrightarrow \mathbb{R}^{n}$ such that $\operatorname{Fix}\left(\left.f\right|_{W}\right)$ is a finite subset of hyperbolic fixed points,

$$
i_{\mathbb{R}^{n}}(f, W)=\sum_{a \in \operatorname{Fix}\left(\left.f\right|_{W}\right)} \operatorname{sign}(J(\operatorname{Id}-f)(a))
$$

where $J$ denotes the Jacobian determinant. Then each attracting fixed point contributes +1 in the above sum.

In this paper we show that the fixed point index in the hyperspace neglects all compact invariant sets that are not attractors.

The next section of this paper will be devoted to presenting the main properties of the fixed compact index of a compact invariant isolated set. Some examples of the consequences of our main result (Theorem 6) are the following corollaries.

Corollary 1. Let $f: U \subset X \longrightarrow X$ be a semidynamical system. A continuum isolated invariant set $K$ is an attractor if and only if $I_{X}(K, f)=1$.

Corollary 2. Let $f: U \subset X \longrightarrow X$ be a semidynamical system. Let $W$ be an open set such that $\mathrm{cl}(W)$ is an isolating neighbourhood with respect to $f$. Then the attracting part of $\operatorname{Inv}(\mathrm{cl}(W), f)$ is non-empty if and only if $i_{2^{x}}\left(\left.2^{f}\right|_{2} w, 2^{W}\right) \neq 0$. In particular, $i_{2} x\left(\left.2^{f}\right|_{2^{W}}, 2^{W}\right) \neq 0$ implies that $W$ contains an attractor. If $\operatorname{Inv}(\mathrm{cl}(W), f)$ is minimal, then $i_{2} x\left(\left.2^{f}\right|_{2} w, 2^{W}\right) \neq 0$ if and only if $\operatorname{Inv}(\mathrm{cl}(W), f)$ is an attractor.

Corollary 3. Let $f: U \subset X \longrightarrow X$ be a semidynamical system. Let $W \subset X$ be an open subset, such that $\mathrm{cl}(W)$ is an isolating neighbourhood with respect to $f$. If $i_{2^{x}}\left(\left.2^{f}\right|_{2^{W}}, 2^{W}\right)=2^{r}-1$ then $2^{f}$ has at least $r$ periodic attracting orbits in $2^{W}$.

COROLlary 4. Let $f: U \subset \mathbb{R} \longrightarrow \mathbb{R}$ be a semidynamical system. Let $K$ be a compact isolated invariant set with respect to $f$. If $I_{\mathbb{R}}(K, f)=2^{r}-1$ then $K$ contains at least $r$ periodic orbits.

Corollary 5. Let $f: U \subset X \longrightarrow X$ be a semidynamical system. Let $K$ be a compact isolated invariant set with respect to $f$ whose components are cell-like. If $I_{X}(K, f)=2^{r}-1$ then $K$ contains at least $r$ periodic orbits. 
COROLlary 6. Let $f: U \subset X \longrightarrow X$ be a semidynamical system. Let $W \subset X$ be an open subset, such that $\mathrm{cl}(W)$ is an isolating neighbourhood of a compact invariant set $K$. If the shape index of $(K, f)$ is the shape of a pointed space $(Y, *)($ see $[\mathbf{1 4}, 19])$ and $i_{2^{x}}\left(\left.2^{f}\right|_{2^{W}}, 2^{W}\right)=I_{X}(K, f)=2^{r}-1$ then $Y$ has at least $r+1$ components.

\section{Properties of the index and the main results}

First of all we will state the main properties, analogous to the Conley index, of our index. All of them follow from the corresponding properties of the fixed point index. Only the additivity property is not obvious.

Proposition 1 (Ważewski property). $\quad I_{X}(K, f) \neq 0$ implies that $K \neq \varnothing$.

Proposition 2 (additivity property). Let $K$ be a compact isolated invariant set. Assume that $K$ is a disjoint sum of two compact invariant isolated sets $K_{1}$ and $K_{2}$. Then,

$$
I_{X}(K, f)=I_{X}\left(K_{1}, f\right)+I_{X}\left(K_{2}, f\right)+I_{X}\left(K_{1}, f\right) I_{X}\left(K_{2}, f\right) .
$$

Proof. Take $N=N_{1} \cup N_{2}$, an isolating neighbourhood of $K$ such that $N_{1} \cap$ $N_{2}=\varnothing$ and $N_{j}$ is an isolating neighbourhood for $K_{j}, j=1,2$.

Choose open neighbourhoods $U_{1} \subset N_{1}$ and $U_{2} \subset N_{2}$ of $K_{1}$ and $K_{2}$ respectively such that $f\left(U_{i}\right) \cap U_{j}=\varnothing$ if $i \neq j$.

Then,

and

$$
I_{X}(K, f)=i_{2^{X}}\left(\left.2^{f}\right|_{2^{U}{ }_{1} \cup U_{2}}, 2^{U_{1} \cup U_{2}}\right)
$$

$$
I_{X}\left(K_{i}, f\right)=i_{2^{X}}\left(\left.2^{f}\right|_{2^{U}}, 2^{U_{i}}\right), \quad i \in\{1,2\} \text {. }
$$

Let $W_{1}=2^{U_{1} \cup U_{2}}, W_{2}=2^{U_{1}} \vee 2^{U_{2}} \vee\left(2^{U_{1}} \times 2^{U_{2}}\right), X_{1}=2^{X}$ and $X_{2}=\left(2^{X}\right)_{1} \vee\left(2^{X}\right)_{2} \vee$ $\left(2^{X} \times 2^{X}\right)$.

Consider the map $i_{*}: W_{1} \longrightarrow W_{2}$ defined in the following way: if $K_{j}^{\prime} \subset U_{j}$, $i_{*}\left(K_{j}^{\prime}\right)=K_{j}^{\prime}, j=1,2$; on the other hand, for $K^{\prime} \in W_{1}$ such that $K^{\prime} \cap U_{1}=K_{1}^{\prime} \neq \varnothing$ and $K^{\prime} \cap U_{2}=K_{2}^{\prime} \neq \varnothing, i_{*}\left(K^{\prime}\right)=\left(K_{1}^{\prime}, K_{2}^{\prime}\right) \in 2^{U_{1}} \times 2^{U_{2}} \subset W_{2}$.

It is easy to see that $i_{*}$ is a homeomorphism.

Let

and

$$
F_{*}=2^{f} \vee 2^{f} \vee\left(2^{f} \times 2^{f}\right): W_{2} \longrightarrow X_{2}
$$

$$
j_{*}: X_{2} \longrightarrow X_{1}
$$

be defined by $j_{*}\left(K_{j}^{\prime}\right)=K_{j}^{\prime}$ if $K_{j}^{\prime} \in\left(2^{X}\right)_{j}$ and $j_{*}\left(K_{1}^{\prime}, K_{2}^{\prime}\right)=K_{1}^{\prime} \cup K_{2}^{\prime}$ for $\left(K_{1}^{\prime}, K_{2}^{\prime}\right) \in 2^{X} \times 2^{X}$. It follows that $2^{f}=j_{*} \circ F_{*} \circ i_{*}$.

Now denote

$$
f_{1}=i \circ i_{*}: W_{1} \longrightarrow X_{2}
$$

where $i: W_{2} \longrightarrow X_{2}$ is the inclusion and

$$
f_{2}=2^{f} \circ i_{*}^{-1}: W_{2} \longrightarrow X_{1} .
$$

Then, $S=\left\{x \in f_{1}^{-1}\left(W_{2}\right):\left(f_{2} \circ f_{1}\right)(x)=x\right\}=\left\{x \in W_{1}: 2^{f}(x)=x\right\}$ is a compact set.

Using the commutativity property of the fixed point index,

$$
\begin{aligned}
i_{2} X\left(2^{f}, 2^{U_{1} \cup U_{2}}\right)= & i_{X_{1}}\left(f_{2} \circ f_{1}, f_{1}^{-1}\left(W_{2}\right)\right)=i_{X_{2}}\left(f_{1} \circ f_{2}, f_{2}^{-1}\left(W_{1}\right)\right) \\
& =i_{\left(2^{X}\right)_{1} \vee\left(2^{X}\right)_{2} \vee\left(2^{X}{ }^{X} 2^{X}\right)}\left(i \circ i_{*} \circ 2^{f} \circ i_{*}^{-1},\left(2^{f} \circ i_{*}^{-1}\right)^{-1}\left(2^{U_{1} \cup U_{2}}\right)\right),
\end{aligned}
$$

since $2^{f}=j_{*} \circ F_{*} \circ i_{*}$

$$
\left.i \circ i_{*} \circ 2^{f} \circ i_{*}^{-1}\right|_{i_{*}\left(\left(2^{f}\right)^{-1}\left(W_{1}\right)\right)}=\left.i \circ i_{*} \circ j_{*} \circ F_{*}\right|_{F_{*}^{-1}\left(j_{*}^{-1}\left(W_{1}\right)\right)} .
$$


On the other hand,

Thus,

$$
\left.i \circ i_{*} \circ j_{*} \circ F_{*}\right|_{F_{*}^{-1}\left(j_{*}^{-1}\left(W_{1}\right)\right)}=\left.F_{*}\right|_{F_{*}^{-1}\left(j_{*}^{-1}\left(W_{1}\right)\right)} .
$$

$$
\begin{aligned}
& i_{\left(2^{X}\right)_{1} \vee\left(2^{X}\right)_{2} \vee\left(2^{X} \times 2^{X}\right)}\left(i \circ i_{*} \circ 2^{f} \circ i_{*}^{-1},\left(2^{f} \circ i_{*}^{-1}\right)^{-1}\left(2^{U_{1} \cup U_{2}}\right)\right) \\
& =i_{\left(2^{X}\right)_{1} \vee\left(2^{X}\right)_{2} \vee\left(2^{X} \times 2^{X}\right)}\left(i \circ i_{*} \circ j_{*} \circ F_{*}, F_{*}^{-1}\left(j_{*}^{-1}\left(W_{1}\right)\right)\right) \\
& =i_{\left(2^{X}\right)_{1} \vee\left(2^{X}\right)_{2} \vee\left(2^{X} \times 2^{X}\right)}\left(F_{*}, F_{*}^{-1}\left(j_{*}^{-1}\left(W_{1}\right)\right)\right) \\
& =i_{X_{2}}\left(F_{*}, F_{*}^{-1}\left(j_{*}^{-1}\left(W_{1}\right)\right)\right)=i_{X_{2}}\left(F_{*}, W_{2}\right) \\
& =i_{X_{2}}\left(\left.F_{*}\right|_{2^{U}}, 2^{U_{1}}\right)+i_{X_{2}}\left(\left.F_{*}\right|_{2^{U_{2}}}, 2^{U_{2}}\right)+i_{X_{2}}\left(\left.F_{*}\right|_{2^{U_{1 \times 2} U^{U}}}, 2^{U_{1}} \times 2^{U_{2}}\right) \\
& =i_{2^{x}}\left(\left.2^{f}\right|_{2^{U}}, 2^{U_{1}}\right)+i_{2^{X}}\left(\left.2^{f}\right|_{2^{U_{2}}}, 2^{U_{2}}\right)+i_{2^{X} \times 2^{X}}\left(2^{f} \times\left. 2^{f}\right|_{2^{U}{ }_{1 \times 2} U_{2}}, 2^{U_{1}} \times 2^{U_{2}}\right) \\
& =i_{2^{X}}\left(\left.2^{f}\right|_{2^{U}}, 2^{U_{1}}\right)+i_{2^{X}}\left(\left.2^{f}\right|_{2^{U_{2}}}, 2^{U_{2}}\right)+i_{2^{x}}\left(\left.2^{f}\right|_{2^{U}}, 2^{U_{1}}\right) i_{2^{X}}\left(\left.2^{f}\right|_{2^{U}}, 2^{U_{2}}\right) \text {. }
\end{aligned}
$$

Proposition 3 (commutativity property). Let $X, Y$ be locally compact metric ANRs. Let

be locally defined maps.

$$
\begin{aligned}
& \varphi: U \subset X \longrightarrow Y, \\
& \psi: V \subset Y \longrightarrow X
\end{aligned}
$$

Take $f=\psi \circ \varphi$ and $g=\varphi \circ \psi$. If $K \subset X$ is an isolated invariant set with respect to $f$ then $\varphi(K)$ is an isolated invariant set with respect to $g$ and $I_{X}(K, f)=I_{Y}(\varphi(K), g)$.

Corollary 7. Let $f: U \subset X \longrightarrow X$ be a locally defined map. Assume that $f(X) \subset Y$ where $Y \subset X$ is a locally compact ANR. If $K$ is a compact isolated invariant set with respect to $f$ then $K$ is an isolated invariant set with respect to $\left.f\right|_{Y}$ and $I_{X}(K, f)=I_{Y}\left(K,\left.f\right|_{Y}\right)$.

Proposition 4 (homotopy property). Let $f: U \times \Lambda \longrightarrow X$ be a map such that $U$ is an open subset of $X$ and $\Lambda \subset \mathbb{R}$ is a compact interval. Assume that $N$ is an isolating neighbourhood for each partial map $f_{\lambda}: U \longrightarrow X$. Then $I_{X}\left(\operatorname{Inv}\left(N, f_{\lambda}\right), f_{\lambda}\right)$ does not depend on $\lambda \in \Lambda$.

The next result is a first approximation to obtain the full meaning of our index. The proof is based on the following theorem due to H. Steinlein; see [18, 22] for details.

THEOREM 2. Let $f: U \subset X \longrightarrow X$ be a locally defined map. Let $H \subset U$ be an open set such that $f^{m}$ is defined on $H$ for $m=p^{t}$ with $p$ prime. Assume that

$$
\Sigma=\left\{x \in H: f^{m}(x)=x\right\}
$$

is compact, $f(\Sigma) \subset \Sigma$ and $f$ is compact in some neighbourhood of $\Sigma$. Then

$$
i_{X}\left(f^{m}, H\right) \equiv i_{X}(f, H)(\bmod p) .
$$

THEOREM 3. Let $f: U \subset X \longrightarrow X$ be a locally defined map. If $K \subset U$ is a compact connected attractor for $f$ then $I_{X}(K, f)=1$.

Proof. Take a connected open set $U_{0}$ as in the above definition. From Theorem $1,2^{U_{0}}$ is an AR.

Consider a prime $q$ and $t$ such that $p=q^{t} \geqslant m\left(U_{0}\right)$, then $\left(2^{f}\right)^{p}=2^{f^{p}}: 2^{U_{0}} \longrightarrow 2^{U_{0}}$. 
It is easy to see that we are in the hypotheses of Steinlein's theorem for $H=2^{U_{0}}$. On the other hand,

$$
i_{2^{x}} x\left(\left(2^{f}\right)^{p}, 2^{U_{0}}\right)=i_{2^{U_{0}}}\left(\left(2^{f}\right)^{p}, 2^{U_{0}}\right)=\Lambda\left(\left(2^{f}\right)^{p}\right)=1
$$

where $\Lambda\left(\left(2^{f}\right)^{p}\right)$ denotes the Lefschetz number of $\left(2^{f}\right)^{p}$ (see [17]).

Therefore, for every prime $q$ we have

$$
1=i_{2^{x}}\left(\left(2^{f}\right)^{p}, 2^{U_{0}}\right) \equiv i_{2^{x}}\left(2^{f}, 2^{U_{0}}\right)=I_{X}(K, f)(\bmod q) .
$$

Then $I_{X}(K, f)=1$.

From Theorem 3 and the additivity property we obtain the next corollary.

Corollary 8. Let $f: U \subset X \longrightarrow X$ be a locally defined map. Let $K$ be a compact isolated invariant set with respect to $f$ which is disjoint sum of $n$ connected attractors $K=K_{1} \cup K_{2} \cup \ldots \cup K_{n}$. Then $I_{X}(K, f)=2^{n}-1$.

Let $K$ be a compact invariant set with respect to the semidynamical system $f: U \subset X \longrightarrow X$. Assume that $K$ has a finite number of components $K_{1}, K_{2}, \ldots, K_{p}$. Since $f(K)=K, f$ produces a permutation of the elements of this decomposition of $K$, we can order the components of $K$ in the following way:

$$
K=K_{1,1} \cup K_{1,2} \cup \ldots \cup K_{1, k_{1}} \cup K_{2,1} \cup K_{2,2} \cup \ldots \cup K_{2, k_{2}} \cup \ldots \cup K_{r, 1} \cup K_{r, 2} \cup \ldots \cup K_{r, k_{r}}
$$

where $k_{1}+k_{2}+\ldots+k_{r}=p$, and for $i \in\{1,2, \ldots, r\}$ and $j \in\left\{1,2, \ldots, k_{i}-1\right\}$ we have $f\left(K_{i, j}\right)=K_{i, j+1}$ and $f\left(K_{i, k_{i}}\right)=K_{i, 1}$.

Definition 3. In the above situation we say that $f$ decomposes $K$ in $r$ cycles. For each $i \in\{1,2, \ldots, r\}$, the corresponding $k_{i}$ is called the length of the cycle $i$.

Remark 5. Assume $K_{0}$ to be a compact attractor of the semidynamical system $f: U \subset X \longrightarrow X$. Since $X$ is locally connected, $K_{0}$ has a finite number of components. Indeed, take an isolating neighbourhood $N=N_{1} \cup \ldots \cup N_{p}$ for $K_{0}$ where $N_{i}$ is connected and $N_{i} \cap K_{0} \neq \varnothing$ for every $i=1, \ldots, p$. Consider $n_{0} \in \mathbb{N}$ such that for each $n \geqslant n_{0}, f^{n}(N) \subset N$. It is clear that for every $i \in\{1, \ldots, p\}$ there exists $p(i) \in\{1, \ldots, p\}$ such that $\left(f^{n_{0}}\right)^{p(i)}\left(N_{i}\right) \subset N_{i}$. If $K_{0}^{i}=K_{0} \cap N_{i}$ then $\left(f^{n_{0}}\right)^{p(i)}\left(K_{0}^{i}\right)=K_{0}^{i}$ and $K_{0}^{i} \subset$ $\operatorname{Inv}\left(N_{i},\left(f^{n_{0}}\right)^{p(i)}\right) \subset N_{i}$. On the other hand, if $x \in \operatorname{Inv}\left(N_{i},\left(f^{n_{0}}\right)^{p(i)}\right)$, since $f^{n_{0}}(N) \subset N$, we have $x \in \operatorname{Inv}(N, f)=K_{0}$. Therefore $x \in K_{0}^{i}, \quad K_{0}^{i}=\operatorname{Inv}\left(N_{i},\left(f^{n_{0}}\right)^{p(i)}\right)$ and $K_{0}^{i}$ is connected.

In general, it is known that there are non-locally connected, connected phase spaces $X$ that admit a global attractor with infinitely many connected components, see [9].

Theorem 4. Let $f: U \subset X \longrightarrow X$ be a semidynamical system. Let $K$ be an attractor. Then

$$
I_{X}(K, f)=2^{r}-1
$$

where $r$ is the number of cycles of $K$.

Proof. Consider the cycles decomposition of $K$

$$
K=K_{1,1} \cup K_{1,2} \cup \ldots \cup K_{1, k_{1}} \cup K_{2,1} \cup K_{2,2} \cup \ldots \cup K_{2, k_{2}} \cup \ldots \cup K_{r, 1} \cup K_{r, 2} \cup \ldots \cup K_{r, k_{r}}
$$

where for $i \in\{1,2, \ldots, r\}$ and $j \in\left\{1,2, \ldots, k_{i}-1\right\}, f\left(K_{i, j}\right)=K_{i, j+1}$ and $f\left(K_{i, k_{i}}\right)=K_{i, 1}$. 
From the additivity property it is enough to show that

$$
I_{X}\left(K_{i, 1} \cup K_{i, 2} \cup \ldots \cup K_{i, k_{i}}, f\right)=1
$$

for any $i \in\{1,2, \ldots, r\}$.

Then we can assume that $K$ has a single cycle $K=K_{1} \cup K_{2} \cup \ldots \cup K_{k}$.

Consider an isolating neighbourhood $N$ of $K$ as in Remark 5 and an open $W \subset N$ containing $K . W$ is union of open components, $W=W_{1} \cup W_{2} \cup \ldots \cup W_{k}$, and

where

$$
2^{W}=\bigcup_{A \subset\{1, \ldots, k\}} W_{A}
$$

$$
W_{A}=\left\{C \in 2^{W}: C \cap W_{j} \neq \varnothing \text { for every } j \in A \text { and } C \cap W_{j}=\varnothing \text { if } j \notin A\right\} .
$$
ARs.

Since $W_{A}$ is homeomorphic to the product $\prod_{j \in A} 2^{W_{j}}$, the components of $2^{W}$ are

Now let $m>k$ be any prime such that $f^{m}(W) \subset W$, then

$$
i_{2^{x}}\left(2^{f^{m}}, 2^{W}\right)=i_{2} w\left(2^{f^{m}}, 2^{W}\right)=\Lambda\left(2^{f^{m}}\right) .
$$

In order to compute $\Lambda\left(2^{f^{m}}\right)$ we just have to pay attention to

$$
H_{0}\left(2^{W}\right)=\mathbb{Q} \oplus \ldots \oplus \mathbb{Q} \quad \text { with } 2^{k}-1 \text { generators. }
$$

Consider the generators of $H_{0}\left(2^{W}\right)$ corresponding to the components of $2^{W}$ and assume that

where $p<k$.

$$
f^{m}\left(K_{i_{1}} \cup \ldots \cup K_{i_{p}}\right)=K_{i_{1}} \cup \ldots \cup K_{i_{p}}
$$

Then $\left\{K_{i_{1}}, \ldots, K_{i_{p}}\right\}$ is a cycle or union of cycles for $f^{m}$ and

$$
p<k, \quad p m=n k
$$

for some $n \in \mathbb{N}$.

Since $m$ is prime, $m>k$, we have $n=n_{1} m$ and $p=n_{1} k$. Then $n_{1}=1, n=m$ and $p=k$ which is a contradiction.

Consequently

$$
\left\{K_{i_{1}}, \ldots, K_{i_{p}}\right\}=\left\{K_{1}, \ldots, K_{k}\right\}
$$

and just the generator of $H_{0}\left(W_{\{1, \ldots, k\}}\right)$ produces a non-trivial number in the trace of the matrix of $\left(2^{f^{m}}\right) *$ which has the form

$$
\left(\begin{array}{llll}
0 & & & \\
& \ddots & & \\
& & 0 & \\
& & & 1
\end{array}\right) .
$$

Then from Theorem 2,

$$
i_{2^{X}}\left(2^{f^{m}}, 2^{W}\right)=i_{2^{w}}\left(2^{f^{m}}, 2^{W}\right)=\Lambda\left(2^{f^{m}}\right)=(-1)^{0} \operatorname{tr}\left(2^{f^{m}}\right)^{*}=1 \equiv I_{X}(K, f)(\bmod m) .
$$

Since $m>k$ is arbitrary, we have $I_{X}(K, f)=1$.

In order to compute the index in more general cases we will recall the notion of index pair which is basic to construct the Conley index. A certain class of index pairs, regular index pairs, will be useful. 
Definition 4 [12]. Let $N$ be a compact isolating neighbourhood with respect to $f: U \subset X \longrightarrow X$ and $K=\operatorname{Inv}(N)$. Let $\left(P_{1}, P_{2}\right)$ be a pair of closed subsets of $N$ such that $P_{2} \subset P_{1}$. The pair $\left(P_{1}, P_{2}\right)$ is called an index pair for $K$ if the following conditions are satisfied:

(i) $P_{1} \cap f\left(P_{2}\right) \subset P_{2}$;

(ii) $f\left(P_{1} \backslash P_{2}\right) \subset P_{1}$;

(iii) $K=\operatorname{Inv}\left(P_{1} \backslash P_{2}, f\right) \subset \operatorname{Int}\left(P_{1} \backslash P_{2}\right)$.

We will say that the index pair $\left(P_{1}, P_{2}\right)$ is regular if

(a) there exists a set $V$, open in $P_{1}$, such that $P_{2} \subset V$ and $f\left(V \backslash P_{2}\right) \subset P_{2}$;

(b) $\operatorname{cl}\left(f\left(P_{2}\right) \backslash P_{1}\right) \cap \operatorname{cl}\left(P_{1} \backslash P_{2}\right)=\varnothing$.

Let $f: U \subset X \longrightarrow X$ be a semidynamical system and consider the induced semidynamical system $2^{f}: 2^{U} \subset 2^{X} \longrightarrow 2^{X}$.

If $K$ is a compact invariant isolated set with respect to $f$ and $N$ is an isolating neighbourhood for $K$ then $2^{N}$ is an isolating neighbourhood for $\operatorname{Inv}\left(2^{N}, 2^{f}\right)=2^{K}$.

The next proposition can be easily checked.

Proposition 5. Let $f: U \subset X \longrightarrow X$ be a semidynamical system. Let $P=\left(P_{1}, P_{2}\right)$ be a compact pair of subsets of $X$.

Let

$$
\bigcap P_{2}=\left\{K \in 2^{P_{1}}: K \cap P_{2} \neq \varnothing\right\} .
$$

If $\left(P_{1}, P_{2}\right)$ is an index pair (regular index pair) for a compact invariant isolated set $K$ then $\left(2^{P_{1}}, \bigcap P_{2}\right)$ is an index pair (regular index pair) for $2^{K}$.

Proposition 6. Under the assumptions of Proposition 5, if $P_{1}$ is locally continuumconnected then $2^{P_{1}}$ and $\bigcap P_{2}$ are finite sums of ARs.

Proof. For $2^{P_{1}}$ apply the arguments of Theorem 4 for $2^{W}$.

The case of $\bigcap P_{2}$ is based on the fact that $\bigcap P_{2}$ is a growth hyperspace of $P_{1}$.

Indeed, if $P_{1}=P_{11} \cup P_{12} \cup \ldots \cup P_{1 k}, P_{1 j}$ connected for every $j \in\{1,2, \ldots, k\}$ then

$$
2^{P_{1}}=\bigcup_{A \subset\{1, \ldots, k\}} P_{1 A}
$$

where

$$
P_{1 A}=\left\{C \in 2^{P_{1}}: C \cap P_{1 j} \neq \varnothing \text { for every } j \in A \text { and } C \cap P_{1 j}=\varnothing \text { if } j \notin A\right\}
$$

and

$$
\bigcap P_{2}=\bigcup_{A \subset\{1, \ldots, k\}} P_{1 A} \cap \bigcap P_{2} .
$$

Let $K_{0}$ be a compact isolated invariant set, $P=\left(P_{1}, P_{2}\right)$ be an index pair for $K_{0}$ such that $P_{1}$ is a finite sum of connected components.

We can order them as follows,

where

$$
P_{1}=P_{1}^{1} \cup \ldots \cup P_{1}^{k_{0}} \cup \ldots \cup P_{1}^{k} \cup \ldots \cup P_{1}^{l}
$$

(a) $P_{1}^{j} \cap K_{0} \neq \varnothing$ and $P_{1}^{j} \cap P_{2}=\varnothing$ if $j \in\left\{1,2, \ldots, k_{0}\right\}$;

(b) $P_{1}^{j} \cap P_{2} \neq \varnothing$ if $j \geqslant k$.

LEMMA 1. Under the above assumptions we have the following:

(1) If $x \in P_{1}^{i}, k \leqslant i \leqslant l$ and $f(x) \in P_{1}^{j}$ then $k \leqslant j \leqslant l$.

(2) If $x \in P_{1}^{i}, 1 \leqslant i \leqslant k_{0}$ then $f(x) \in P_{1}^{j}, 1 \leqslant j \leqslant k_{0}$. 
Proof. (1) If $f\left(P_{1}^{i}\right) \subset P_{1}^{j}$ then $f(y) \in P_{1}^{j} \cap P_{2}$ for any $y \in P_{1}^{i} \cap P_{2}$.

If $P_{1}^{i} \backslash f^{-1}\left(P_{1}^{j}\right) \neq \varnothing$ since $P_{1}^{i}$ is connected there is $p \in \operatorname{cl}_{P_{1}^{i}}\left(P_{1}^{i} \backslash f^{-1}\left(P_{1}^{j}\right)\right) \cap\left(f^{-1}\right.$ $\left.\left(P_{1}^{j}\right) \cap P_{1}^{i}\right)$.

It is easy to see that $p \in P_{2} \cap f^{-1}\left(P_{1}^{j}\right)$. Then $f(p) \in P_{2} \cap P_{1}^{j}$.

(2) Assume $P_{1}^{i_{0}}, 1 \leqslant i_{0} \leqslant k_{0}$, such that $f\left(P_{1}^{i_{0}}\right) \subset P_{1}^{j_{0}}$ with $j_{0} \in\{k, \ldots, l\}$. From (1) there is $i_{1} \leqslant k_{0}$ such that $\left(P_{1}^{i_{1}} \cap K_{0}\right) \cap f\left(P_{1}\right)=\varnothing$. This is a contradiction.

REMARK 6. In the hypotheses of Lemma 1,

$$
\left(P_{1}^{\prime}, P_{2}^{\prime}\right)=\left(P_{1}^{1} \cup \ldots \cup P_{1}^{k_{0}} \cup P_{1}^{k} \cup \ldots \cup P_{1}^{l}, P_{2}\right)
$$

is also an index pair (regular if so is $\left.\left(P_{1}, P_{2}\right)\right)$,

and

$$
K_{0}=\left[K_{0} \cap\left(P_{1}^{1} \cup \ldots \cup P_{1}^{k_{0}}\right)\right] \cup\left[K_{0} \cap\left(P_{1}^{k} \cup \ldots \cup P_{1}^{l}\right)\right]
$$

$$
\begin{aligned}
f\left(K_{0} \cap\left(P_{1}^{1} \cup \ldots \cup P_{1}^{k_{0}}\right)\right) & =K_{0} \cap\left(P_{1}^{1} \cup \ldots \cup P_{1}^{k_{0}}\right), \\
f\left(K_{0} \cap\left(P_{1}^{k} \cup \ldots \cup P_{1}^{l}\right)\right) & =K_{0} \cap\left(P_{1}^{k} \cup \ldots \cup P_{1}^{l}\right) .
\end{aligned}
$$

Denote $K_{0} \cap\left(P_{1}^{1} \cup \ldots \cup P_{1}^{k_{0}}\right)=K_{0}^{\mathrm{A}}, K_{0} \cap\left(P_{1}^{k} \cup \ldots \cup P_{1}^{l}\right)=K_{0} \backslash K_{0}^{\mathrm{A}}$.

$K_{0}^{\mathrm{A}}$ and $K_{0} \backslash K_{0}^{\mathrm{A}}$ are compact isolated invariant sets. We will call $K_{0}^{\mathrm{A}}$ the attracting part of $K_{0}$.

TheORem 5. Let $f: X \longrightarrow X$ be a semidynamical system. Assume that $2^{f}$ is of compact attraction. Let $K_{0}$ be a compact isolated invariant set with respect to $f$ that admits a regular index pair $P=\left(P_{1}, P_{2}\right)$ such that $P_{1}$ is locally continuum-connected.

Then,

$$
I_{X}\left(K_{0}, f\right)=I_{X}\left(K_{0}^{\mathrm{A}}, f\right)=2^{q}-1
$$

where $q$ is the number of cycles of $K_{0}^{\mathrm{A}}$.

Proof. We maintain the notation of Remark 6 . Using the additivity property it is enough to show that $I_{X}\left(K_{0} \backslash K_{0}^{\mathrm{A}}, f\right)=0$.

$P^{\prime}=\left(P_{1}^{\prime}, P_{2}^{\prime}\right)=\left(P_{1}^{k} \cup \ldots \cup P_{1}^{l}, P_{2}\right)$ is a regular index pair for $K_{0} \backslash K_{0}^{\mathrm{A}}$.

Then $\left(2^{P_{1}^{\prime}}, \bigcap P_{2}^{\prime}\right)$ is a regular index pair associated with the compact invariant isolated set $2^{K_{0} \backslash K_{0}^{A}}$ with respect to $2^{f}: 2^{U} \longrightarrow 2^{X}$.

$2^{P_{1}^{\prime}}$ and $\bigcap P_{2}^{\prime}$ are compact ANRs and finite sums of ARs.

Consider the singular homology sequence of the pair $\left(2^{P_{1}^{\prime}}, \bigcap P_{2}^{\prime}\right)$

$$
\begin{aligned}
& \ldots \longrightarrow H_{p}\left(\bigcap P_{2}^{\prime}\right) \longrightarrow H_{p}\left(2^{P_{1}^{\prime}}\right) \longrightarrow H_{p}\left(2^{P_{1}^{\prime}}, \bigcap P_{2}^{\prime}\right) \longrightarrow H_{p-1}\left(\bigcap P_{2}^{\prime}\right) \longrightarrow \\
& \ldots \longrightarrow H_{1}\left(\bigcap P_{1}^{\prime}\right) \longrightarrow H_{1}\left(2^{P_{1}^{\prime}}\right) \longrightarrow H_{1}\left(2^{P_{1}^{\prime}}, \bigcap P_{2}^{\prime}\right) \\
& \longrightarrow H_{0}\left(\bigcap P_{2}^{\prime}\right) \longrightarrow H_{0}\left(2^{P_{1}^{\prime}}\right) \longrightarrow H_{0}\left(2^{P_{1}^{\prime}}, \bigcap P_{2}^{\prime}\right) \longrightarrow 0 .
\end{aligned}
$$

Since $H_{p}\left(2^{P_{1}^{\prime}}\right)=H_{p}\left(\bigcap P_{2}^{\prime}\right)=0$ for every $p \geqslant 1$, we have $H_{p}\left(2^{P_{1}^{\prime}}, \bigcap P_{2}^{\prime}\right)=0$ for every $p>1$.

Another point of view is that $2^{P_{1}^{\prime}} / \bigcap P_{2}^{\prime}$ is an AR because it is a pointed sum of ARs, then $0=H_{1}\left(2^{P_{1}^{\prime}} / \bigcap P_{2}^{\prime}, *\right) \equiv H_{1}\left(2^{P_{1}^{\prime}}, \bigcap P_{2}^{\prime}\right)$.

Analogously $0=H_{0}\left(2^{P_{1}^{\prime}} / \bigcap P_{2}^{\prime}\right) \equiv H_{0}\left(2^{P_{1}^{\prime}} / \bigcap P_{2}^{\prime}, *\right) \equiv H_{0}\left(2^{P_{1}^{\prime}}, \bigcap P_{2}^{\prime}\right)$.

Now, from [12, Theorem 4],

$$
I_{X}\left(K_{0} \backslash K_{0}^{\mathrm{A}}, f\right)=i_{2} x\left(2^{f}, \operatorname{int}\left(2^{P_{1}^{\prime}} \backslash \bigcap P_{2}^{\prime}\right)\right)=\Lambda\left(I_{2^{P_{1}^{\prime}}, \bigcap P_{2}^{\prime}}\right)=0 .
$$

If $Q=\prod_{n=1}^{\infty}[0,1 / n]$ denotes the Hilbert cube, we have the following corollary. 
Corollary 9. Let $f: U \subset Q \longrightarrow Q$ be a semidynamical system. Let $K_{0}$ be a compact isolated invariant set with respect to $f$ that admits a regular index pair $P=$ $\left(P_{1}, P_{2}\right)$ such that $P_{1}$ is locally continuum-connected.

Then,

$$
I_{X}\left(K_{0}, f\right)=I_{X}\left(K_{0}^{\mathrm{A}}, f\right)=2^{q}-1
$$

where $q$ is the number of cycles of $K_{0}^{\mathrm{A}}$.

In order to give the complete meaning of our index we will show that it is always possible to find regular index pairs in the Hilbert cube. On the other hand, we will also prove, using the commutativity property, that for the computation of the index, for an arbitrary compact isolated invariant set in an arbitrary locally compact ANR, we can always assume that our semidynamical system is defined in $Q$.

We shall extend, to our context, the techniques of [23, Lemma 5.1]. Let $f: Q \longrightarrow Q$ be a continuous map. Given $\epsilon>0$, we consider

$$
\begin{array}{r}
\mathscr{A}(\epsilon)=\left\{A \cap Q, A=\prod_{i=1}^{\infty} A_{i} \text { : for all } i \text { there exists } k \in \mathbb{N}\right. \text { with } \\
\left.A_{i}=k \epsilon \text { or } A_{i}=[k \epsilon,(k+1) \epsilon]\right\} .
\end{array}
$$

Define the following multivalued maps $T_{\epsilon}, F_{\epsilon}: Q \longrightarrow \mathscr{P}(Q)$, by

and

$$
T_{\epsilon}(x)=\bigcup\{A \in \mathscr{A}(\epsilon): x \in A\}
$$

$$
F_{\epsilon}(x)=\left(T_{\epsilon} \circ f \circ T_{\epsilon}\right)(x) .
$$

Lemma 2. Consider $F: I \times Q \times \mathbb{Z} \longrightarrow \mathscr{P}(Q)$ defined as

$$
F(\epsilon, x, n)=F_{\epsilon}^{n}(x) .
$$

Then $F$ is a compact valued upper semicontinuous map and for every $\epsilon \in I$, $F_{\varepsilon}: Q \times \mathbb{Z} \longrightarrow \mathscr{P}(Q)$ is a discrete multivalued dynamical system in the sense of Kaczynski and Mrozek [11].

Proof. First we will prove that $F_{\epsilon}^{1}: Q \longrightarrow \mathscr{P}(Q)$ is a proper compact valued upper semicontinuous map and then it generates a discrete multivalued dynamical system (see [11]).

For any $\epsilon>0$ there exists $n(\epsilon) \in \mathbb{N}$ such that $1 / n(\epsilon) \leqslant \epsilon<1 /(n(\epsilon)-1)$.

Then

$$
T_{\epsilon}(x)=P \times Q \subset \prod_{n=1}^{n(\epsilon)-1}\left[0, \frac{1}{n}\right] \times Q
$$

with $P$ a finite polyhedron. Therefore, for all $x \in Q, T_{\epsilon}(x)$ is a prism (see [2, page 104]).

Analogously for every $x \in Q$,

$$
F_{\epsilon}(x)=\left(P_{1} \cup \ldots \cup P_{k}\right) \times Q \subset \prod_{n=1}^{n(\epsilon)-1}\left[0, \frac{1}{n}\right] \times Q
$$

where $P_{i}$ is a finite polyhedron for every $i=1, \ldots, k$. Moreover since $k$ is finite, for every $x \in Q, F_{\epsilon}(x)$ is a prism.

In particular $T_{\epsilon}$ and $F_{\epsilon}$ are compact valued maps. It is easy to see that both maps are upper semicontinuous.

Then, $F_{\epsilon}^{1}: Q \longrightarrow \mathscr{P}(Q)$ is a compact valued proper upper semicontinuous multivalued map generating a discrete multivalued dynamical system. 
In order to check the upper semicontinuity of $F$, take an open subset $U \subset Q$.

The set

$$
\{(\epsilon, x, n) \in I \times Q \times \mathbb{Z}: F(\epsilon, x, n) \subset U\} \equiv \bigcup_{n \in \mathbb{Z}}\{(\epsilon, x, n) \in I \times Q \times n: F(\epsilon, x, n) \subset U\}
$$

must be an open subset of $I \times Q \times \mathbb{Z}$.

Then it is enough to see that

$$
F^{1}, F^{-1}: I \times Q \longrightarrow \mathscr{P}(Q)
$$

are upper semicontinuous.

The proof for $F^{1}$ is obvious because $T: I \times Q \longrightarrow \mathscr{P}(Q)$, where $T(\epsilon, x)=T_{\epsilon}(x)$, is upper semicontinuous.

On the other hand, for $\left(\epsilon_{0}, x_{0}\right) \in\left\{(\epsilon, x) \in I \times Q: F^{-1}(\epsilon, x) \subset U\right\}$ it follows that

$$
F_{\epsilon}^{-1}(x) \subset U \text { when } \epsilon \rightarrow \epsilon_{0} \text { and } x \rightarrow x_{0} .
$$

Indeed, otherwise there exist sequences $\left\{\epsilon_{n}\right\}_{n}$, with $\epsilon_{n} \rightarrow \epsilon_{0},\left\{x_{n}\right\}_{n} \rightarrow x_{0}$ and $\left\{y_{n}\right\}_{n} \rightarrow y_{0}$, with $y_{n} \notin U$ for every $n$ and such that $y_{n} \in F_{\epsilon_{n}}^{-1}\left(x_{n}\right)$.

Since $x_{n} \in F_{\epsilon_{n}}^{1}\left(y_{n}\right)$ for every $n \in \mathbb{N}$ and $F^{1}$ is upper semicontinuous,

$$
\operatorname{Sup}_{y \in F_{\epsilon_{n}}^{1}\left(y_{n}\right)}\left\{d\left(y, F_{\epsilon_{0}}^{1}\left(y_{0}\right)\right)\right\} \rightarrow 0 \text { if } n \rightarrow \infty .
$$

Consequently, $\quad d\left(x_{0}, F_{\epsilon_{0}}^{1}\left(y_{0}\right)\right) \leqslant d\left(x_{0}, F_{\epsilon_{n}}^{1}\left(y_{n}\right)\right)+\operatorname{Sup}_{y \in F_{n}^{1}\left(y_{n}\right)}\left\{d\left(y, F_{\epsilon_{0}}^{1}\left(y_{0}\right)\right)\right\} \rightarrow 0 \quad$ if $n \rightarrow \infty, x_{0} \in F_{\epsilon_{0}}^{1}\left(y_{0}\right)$ and $y_{0} \in F_{\epsilon_{0}}^{-1}\left(x_{0}\right) \subset U$ which is a contradiction.

Proposition 7. Let $f: Q \longrightarrow Q$ be a semidynamical system. Then, every compact isolated invariant set $K$, with respect to $f$, admits a regular index pair $\left(Q_{1}, Q_{0}\right)$ such that $Q_{1}$ and $Q_{0}$ are prisms.

Proof. The proof is based on that of [23, Lemma 5.1], using Lemma 2 and [11, Lemmas 2.5 and 2.6]. It is enough to follow the construction of [23] with a slight modification of the definition of the $Q_{i}$. Taking

$$
Q_{i}=\bigcup\left\{A \in \mathscr{A}(\epsilon / d): A \subset P_{i}, A=P \times Q \subset \prod_{n=1}^{n(\epsilon / d)-1}\left[0, \frac{1}{n}\right] \times Q\right\}
$$

where $P$ is a finite polyhedron, the result follows.

THEOREM 6. Let $f: U \subset X \longrightarrow X$ be a semidynamical system. Let $K$ be a compact isolated invariant set with respect to $f$. Then

$$
I_{X}(K, f)=I_{X}\left(K^{\mathrm{A}}, f\right)=2^{q}-1
$$

where $q$ is the number of cycles of $K^{\mathrm{A}}$.

Proof. Consider $X$ to be embedded as a closed subset of a normed space $B$. Let $N$ be a compact isolating neighbourhood of $K$. Take a retraction $r: U^{X} \subset B \longrightarrow X$ where $U^{X}$ is an open neighbourhood of $X$ in $B$. From [8], there is a compact ANR $A^{N}$ such that $N \subset A^{N} \subset U^{X}$.

Let $V$ be an open subset of $X$ such that $K \subset V \subset N$ and $f(V) \subset N$.

Define $W=r^{-1}(V) \cap A^{N}$ and consider the map

$$
f_{1}=f \circ r_{\mid W}: W \subset A^{N} \longrightarrow A^{N} .
$$

Using the commutativity property we have $I_{X}(K, f)=I_{A^{N}}\left(K, f_{1}\right)$. 
Now we can assume that $A^{N} \subset Q$. Take a retraction

$$
r_{1}: U^{A^{N}} \subset Q \longrightarrow A^{N}
$$

where $U^{A^{N}}$ is an open neighbourhood of $A^{N}$ in $Q$.

Let $Z=r_{1}^{-1}(W) \subset U^{A^{N}}$ and define

$$
g=f_{1} \circ r_{1 \mid Z}: Z \subset Q \longrightarrow Q .
$$

Using again the commutativity property we have $I_{A^{N}}\left(K, f_{1}\right)=I_{Q}(K, g)$. Then, from Theorem 5 and Proposition 7,

$$
I_{X}(K, f)=I_{Q}(K, g)=I_{Q}\left(K^{\mathrm{A}}, g\right)=2^{q}-1 .
$$

Remark 7. Using Proposition 7 and the proof of Theorem 6 it can be proved that the shape index (see [14]) of any compact isolated invariant set in any locally compact ANR is always the shape of a $P$-like compactum $Y$. More precisely, $Y$ is the inverse limit of a sequence $\left\{\left(P_{n}, g_{n}\right)\right\}_{n \in \mathbb{N}}$ where, for every $n \in \mathbb{N}, P_{n}$ is a single finite polyhedron $P$ and $g_{n}$ is a single continuous map $g: P \longrightarrow P$ (see [20] for a proof).

COROLlary 10 (product property). Let $f: U \subset X \longrightarrow X, g: V \subset Y \longrightarrow Y$ be two semidynamical systems. Let $K_{0}$ and $L_{0}$ be compact isolated invariant sets with respect to $f$ and $g$ respectively.

Then, $K_{0} \times L_{0}$ is a compact isolated invariant set with respect to $f \times g: U \times V \subset$ $X \times Y \longrightarrow X \times Y$ and

$$
I_{X \times Y}\left(K_{0} \times L_{0}, f \times g\right)=2^{p q}-1
$$

where $p$ and $q$ are the number of cycles of $K_{0}^{\mathrm{A}}$ and $L_{0}^{\mathrm{A}}$, respectively.

Now let us take another growth hyperspace of $X$ that we can use to introduce analogous indices.

Let $C_{m}(X)$ be the set of all non-empty compact subsets of $X$ having at most $m$ connected components. It is easy to see that $C_{m}(X)$ is a growth hyperspace of $X$.

If $f: U \subset X \longrightarrow X$ is a semidynamical system, then $f$ induces another one

$$
C_{m}(f): C_{m}(U) \subset C_{m}(X) \longrightarrow C_{m}(X) .
$$

Let $K \subset U$ be a compact isolated invariant set. Let $N$ be any isolating neighbourhood of $K$. Consider an open set $W$ such that $K \subset W \subset N$.

Take $C_{m}(f): C_{m}(W) \longrightarrow C_{m}(X)$. It is clear that $\operatorname{Fix}\left(\left.C_{m}(f)\right|_{C_{m}(W)}\right) \subset C_{m}(K)$.

Then the fixed point index of $C_{m}(f)$ in $C_{m}(X), i_{C_{m}(X)}\left(C_{m}(f), C_{m}(W)\right)$, is well defined.

Definition 5. We define the m-fixed compact index of the pair $(K, f)$ as

$$
I_{X}^{m}(K, f)=i_{C_{m}(X)}\left(\left.C_{m}(f)\right|_{C_{m}(W)}, C_{m}(W)\right) .
$$

REMARK 8. The properties of these indices are similar to the properties of the fixed compact index of the pair $(K, f)$ listed in Propositions 1-4 except for the additivity property. In this case we can obtain the next result for $m=1,2$.

Proposition 8 (additivity property). Let $K$ be a compact isolated invariant set. Assume that $K$ is a disjoint sum of two compact invariant isolated sets $K_{1}$ and $K_{2}$. 
Then,

and

$$
I_{X}^{1}(K, f)=I_{X}^{1}\left(K_{1}, f\right)+I_{X}^{1}\left(K_{2}, f\right)
$$

$$
I_{X}^{2}(K, f)=I_{X}^{2}\left(K_{1}, f\right)+I_{X}^{2}\left(K_{2}, f\right)+I_{X}^{1}\left(K_{1}, f\right) I_{X}^{1}\left(K_{2}, f\right) .
$$

Even though we do not have an additivity property for all $m \in \mathbb{N}$ we can compute those indices to obtain the corresponding results analogous to the $I_{X}(K, f)$.

We now present the main results.

THEOREM 7. Let $f: U \subset X \longrightarrow X$ be a semidynamical system. Let $K$ be a compact attractor.

Then

$$
I_{X}^{m}(K, f)=l_{m}
$$

where $l_{m}$ is the number of unions of cycles of $K$ whose lengths sum $\leqslant m$.

THEOREM 8. Let $f: U \subset X \longrightarrow X$ be a semidynamical system. Let $K$ be a compact isolated invariant set with respect to $f$.

Then,

$$
I_{X}^{m}(K, f)=I_{X}^{m}\left(K^{\mathrm{A}}, f\right)=l_{m}
$$

where $l_{m}$ is the number of unions of cycles of $K^{\mathrm{A}}$ whose lengths sum $\leqslant m$.

REMARK 9. From Theorems 6 and 8, if we have a compact isolated invariant set $K$ with respect to a semidynamical system $f: U \subset X \longrightarrow X$, there is $m_{0} \in \mathbb{N}$ such that $I_{X}^{m}(K, f)=I_{X}(K, f)$ for every $m \geqslant m_{0}$.

Remark 10. Let $f: U \subset X \longrightarrow X$ and $g: V \subset Y \longrightarrow Y$ be two semidynamical systems and $K_{1}$ and $K_{2}$ be compact isolated invariant sets with respect to $f$ and $g$ respectively. Then, $I_{X}^{m}\left(K_{1}, f\right)=I_{Y}^{m}\left(K_{2}, g\right)$ for $m \leqslant r$ if and only if the number of cycles of length $m$, for $m \leqslant r$, of $K_{1}^{\mathrm{A}}$ and $K_{2}^{\mathrm{A}}$ coincide.

Acknowledgements. The authors want to thank M. A. Morón for comments and ideas that have been useful in completing this research. The first author wishes to dedicate this paper to Esther Jiménez for her love and support. The second author wishes to dedicate this paper to his parents and his sister.

\section{References}

1. N. P. Bhatia and G. P. Szegö, Stability theory of dynamical systems (Springer, Berlin, 1970).

2. K. BorsuK, Theory of retracts, Monografie Matematyczne 44 (PWN, Warsaw, 1967).

3. R. F. Brown, The Lefschetz fixed point theorem (Scott, Foresman, Glenview, IL, 1971).

4. C. C. Conley, 'Isolated invariant sets and the Morse index', CBMS 38 (American Mathematical Society, Providence, RI, 1978).

5. D. W. CuRTiS and R. M. SCHORI, ' $2^{X}$ and $C(X)$ are homeomorphic to the Hilbert cube', Bull. Amer. Math. Soc. 80 (1974) 927-931.

6. D. W. CuRTis, 'Hyperspaces of noncompact metric spaces', Compositio Math. 40 (1980) 139-152.

7. A. Dold, 'Fixed point index and fixed point theorem for Euclidean neighbourhood retracts', Topology 4 (1965) 1-8.

8. J. Girolo, 'Approximating compact sets in normed linear spaces', Pacific J. Math. 98 (1992) $81-89$.

9. M. Gobbino and M. Sardella, 'On the connectedness of attractors for dynamical systems', J. Differential Equations 133 (1997) 1-14.

10. S. T. Hu, Theory of retracts (Wayne State University Press, Detroit, 1965).

11. T. KaCZYNSKi and M. MrozeK, 'Stable index pairs for discrete dynamical systems', Canad. Math. Bull. 40 (1997) 448-455. 
12. M. Mrozek, 'Index pairs and the fixed point index for semidynamical systems with discrete time', Fund. Math. 133 (1989) 179-194.

13. M. Mrozek, 'Leray functor and cohomological Conley index for discrete dynamical systems', Trans. Amer. Math. Soc. 318 (1990) 149-178.

14. M. Mrozek, 'Shape index and other indices of Conley type for local maps on locally compact Hausdorff spaces', Fund. Math. 145 (1994) 15-37.

15. M. MrozeK and K. P. Rybakowski, 'A cohomological Conley index for maps on metric spaces', J. Differential Equations 90 (1991) 143-171.

16. N. T. NHU, 'Investigating the ANR-property of metric spaces', Fund. Math. 124 (1984) 243-254.

17. R. D. Nussbaum, 'Generalizing the fixed point index', Math. Ann. 228 (1977) 259-278.

18. R. D. Nussbaum, 'The fixed point index and some applications', Séminaire de Mathématiques Supérieures (Presses de l'Université de Montréal, 1985).

19. J. W. Robbin and D. Salamon, 'Dynamical systems, shape theory and the Conley index', Ergodic Theory Dynam. Systems 8 (1988) 375-393.

20. F. R. Ruiz del Portal and J. M. Salazar, 'Shape index in metric spaces', preprint.

21. E. SPANIER, Algebraic topology (McGraw-Hill, New York, 1966).

22. H. Steinlein, 'Über die verallgemeinerten Fixpunktindizes von Iterierten verdichtender Abbildungen', Manuscripta Math. 8 (1973) 252-266.

23. A. SzymCZaK, 'The Conley index and symbolic dynamics', Topology 35 (1996) 287-299.

24. J. E. West, 'Mapping Hilbert cube manifolds to ANRs: a solution of a conjecture of Borsuk', Ann. of Math. 106 (1977) 1-18.

Departamento de Geometría y Topología

Facultad de CC Matemáticas

Universidad Complutense de Madrid

Madrid 28040

Spain

r_portal@mat.ucm.es 